DOI: $10.20472 /$ IAC.2020.053.015

\author{
KEITA NISHIO \\ Aoyama Gakuin University, Japan \\ TAKASHI KABURAGI \\ International Christian University, Japan \\ TOSHIYUKI MATSUMOTO \\ Aoyama Gakuin University, Japan \\ SATOSHI KUMAGAI \\ Aoyama Gakuin University, Japan \\ YOSUKE KURIHARA \\ Aoyama Gakuin University, Japan
}

\title{
UNOBTRUSIVE CLASSIFICATION SYSTEM FOR MONITORING SCRATCHING TIME BY WIGNER-VILLE DISTRIBUTION
}

\begin{abstract}
:
Patients with dermatitis can stand scratching itchy parts while waking up; hence, monitoring the scratching time unobtrusively during sleep can achieve a more accurate dermatitis diagnosis. Therefore, in this paper, we proposed an unobtrusive scratching motion measurement system. The signal processing for estimating scratching time from output signals from the sensing device was also presented.

This measurement system includes a sensing device that measures the vibrations due to scratching on a bed. As per the device structure, a piezoelectric ceramic is bonded to a metal plate. The sensing device is set between the floor and a bed leg of the head side. When a patient lying on the bed scratches skin, vibrations due to motion propagate to the sensing device. The device outputs signal proportional to the amplitude of the vibrations. To obtain a feature vector, Wigner-Ville distribution and non-negative matrix factorization are applied to the output signal from the sensing device. Applying an optimal threshold to the feature vector, we classified whether the patient scratches the skin at each discrete time.

The validation experiment was conducted with four males. Each subject is asked to scratch the right cheek with his right hand at an arbitrary timing during the 30-s measurement time for one data. We obtained 14 data from four subjects. We aggregated the estimation results in a confusion matrix. To evaluate the system, sensitivity, specificity, positive predictive value (PPV), negative predictive value (NPV), and F value were calculated from the confusion matrix. Due to an initial value dependence on the non-negative matrix factorization, each index was calculated 100 times, and average and standard deviation were obtained. The sensitivity was $0.94 \pm 2.1 \times 10^{\wedge}(-4)$; specificity, $0.94 \pm 2.2 \times 10^{\wedge}(-4) ; \mathrm{PPV}, 0.87 \pm 2.9 \times 10^{\wedge}(-4) ; \mathrm{NPV}, 0.97 \pm 1.4 \times 10^{\wedge}(-4)$; and F-value, $0.90 \pm 2.6 \times 10^{\wedge}(-4)$. These results showed that the proposed measurement system succeeded in estimating the scratching time unobtrusively.
\end{abstract}

\section{Keywords:}

scratching, Wigner-Ville distribution, nonnegative matrix factorization, piezoelectric ceramic 
JEL Classification: 110 